Política y Sociedad

ISSN: $\quad 1130-8001$

ISSN-e: $1988-3129$

\title{
Capitalismo y personalidad: consideraciones sobre los discursos empresariales de la rentabilización del yo a través de la marca personal
}

Luis Enrique Alonso Benito ${ }^{1}$ y Carlos J. Fernández Rodríguez ${ }^{2}$

Recibido: 04-10-2019/ Aceptado: 31-07-2020

Resumen. Los discursos del management contemporáneo tienen en la personalidad del trabajador uno de sus temas más recurrentes. En el contexto de una nueva economía del conocimiento globalizada caracterizada por la creciente competencia en los mercados, las empresas parecen tener la necesidad de contar con trabajadores que sobresalgan por encima de la media, y a los que se afanan por identificar y contratar. Así, las corporaciones van a estar obsesionadas por un significante, el talento, que definirá a esos empleados extraordinarios frente a otros trabajadores menos preparados y adaptados a lo que el mercado demanda. La búsqueda de ese talento tendrá como contrapunto la necesidad de que los propios trabajadores traten de destacarse mediante la construcción de una marca personal que atraiga la atención y contribuya a la construcción de una reputación profesional, lo que les obliga a hacer un marketing continuo de sí mismos. En este artículo, nuestro objetivo es el de descifrar estos discursos del talento y la marca personal con el fin de promover una reflexión sobre la construcción de una personalidad neoliberal, discutiendo sus implicaciones en la emergencia de nuevos discursos justificadores de la desigualdad social.

Palabras clave: empresa; talento; management; personalidad neoliberal; marca personal.

\section{[en] Capitalism and Personality: Remarks about the Management Discourses on Self-profiting through Personal Branding}

\begin{abstract}
One of the themes that recurrently emerges in contemporary management discourses is the issue of the personality of the worker. In the current context of a global knowledge economy with boisterous markets, companies seem to have the need to find out workers who stand above the average, and they actually strive to identify them and hire them. Therefore, firms seem to be obsessed about one specific signifier, talent, which will define these extraordinary employees vis-à-vis less skilled workers who cannot meet the increasing demands of the market. The search for such talent by firms will have, as a counterpoint, the need for the workers themselves to try to stand out by building their own personal brand in order to attract attention and enhance their professional reputation, forcing them to market themselves constantly. In this article, our goal is to decipher those discourses on talent and personal branding in order to promote a wider reflection on the construction of a neoliberal personality. We will also discuss the implications of these discourses in the emergence of new justifications of social inequality.
\end{abstract}

\footnotetext{
1 Universidad Autónoma de Madrid (España).

E-mail: luis.alonso@uam.es

2 Universidad Autónoma de Madrid (España).

E-mail: carlos.fernandez@uam.es
} 
Keywords: firm; talent; management; neoliberal personality; personal branding.

Cómo citar: Alonso Benito, L.E. y C.J. Fernández Rodríguez (2020): “Capitalismo y personalidad: consideraciones sobre los discursos empresariales de la rentabilización del yo a través de la marca personal", Política y Sociedad, 57(2), pp. 521-541.

Sumario. 1. Introducción. 2. Abriendo la caja negra del talento: la razón de un concepto estrictamente empresarial. 3. Marca personal: venderse a sí mismo. 4. La personalidad neoliberal. 5. Conclusión: La justificación de la desigualdad o el racismo de la inteligencia. 6. Bibliografía.

Agradecimientos. Este trabajo ha sido financiado en el contexto del proyecto de investigación del Ministerio de Ciencia, Innovación y Universidades, con referencia PGC2018-097200-B-I00.

"La diferencia de talentos naturales entre personas es en realidad mucho menor de lo que creemos; y las muy diversas habilidades que distinguen a los hombres de diferentes profesiones, una vez que alcanzan la madurez, con mucha frecuencia no son la causa sino el efecto de la división del trabajo".

Adam Smith (1994: 47)

"El racionalismo económico depende para su aparición tanto de la técnica y el derecho racionales como de la capacidad y la disposición de los hombres a determinadas formas de modo de vida práctico-racionales".

Max Weber (2012: 340)

\section{Introducción}

Los discursos sobre la personalidad de las concepciones actuales del management han encontrado un significante central para desplegar a partir de él todo un conjunto de efectos retóricos tan fascinantes como inquietantes. Se trata del significante del talento que, así, se ha convertido en un auténtico cimiento literario para fundamentar, sobre él, los conceptos sagrados (al uso y abuso) en los textos litúrgicos de las ceremonias empresariales que acompañan a los procesos productivos, comerciales y financieros contemporáneos. Talento es, en este contexto, una suerte de significante impreciso, vacío y flotante (Laclau, 2004: 212), pero que permite construir sobre él un inmenso conjunto de nuevos semantemas que tratan de designar, y por tanto de prescribir, la realidad de la empresa y el trabajo en la era neoliberal.

De este modo, conceptos como emprendimiento, marca personal (o emocional), nuevo liderazgo o ingeniería creativa, por solo citar algunos, tienen su referencia última en un uso inspirador de la noción de talento, que sirve para abrir lo que parecía un discurso económico y técnico a un horizonte humanístico. El discurso del management, de esta manera, cada vez se articula más a base de utilizar términos importados del discurso de las artes, la psicología o el consumo cultural, con el fin de ocultar el punto crítico del control sobre la organización y el proceso 
de trabajo. Los repertorios clásicos sobre jerarquización, divisionalización, superficie de control, asignación de tareas o primas de productividad —centrales en el discurso gerencial canónico - se han ido diluyendo en un discurso mucho más borroso, donde se difuminan las barreras instrumentales y se instaura un nuevo género en el que se mezcla la autoayuda con la teoría del caos, la epopeya personal con el relato triunfal, o la inteligencia emocional con el diseño creativo. Todo vale si de lo que se trata es de resaltar el valor de una personalidad arrolladora, que es capaz de poner en marcha - si se deja de complejos sociales, escrúpulos colectivos o frenos institucionales - la deslumbrante tramoya de la maquinaria creativa de la empresa: en esta representación, el papel del talento se convierte en el personaje principal de esta obra de ilusionismo.

En este artículo, nuestro objetivo es el de realizar un análisis y reflexión teórica sobre este concepto de talento y su manifestación corporativa más cercana, como es la noción de marca personal, en coherencia con una fecunda línea de investigación centrada en dar cuenta de las características e implicaciones del discurso gerencial contemporáneo y su extensión en todas las esferas de la sociedad, y que ha generado numerosas publicaciones (ver Alonso y Fernández Rodríguez, 2006, 2013a, 2013b y 2018; Ampudia de Haro, 2019; Fernández Rodríguez, 2007 y 2019; Muñoz-Rodríguez y Santos Ortega, 2017; Revilla, 2017; Serrano Pascual, 2016). Para ello, dividiremos nuestra contribución en cuatro secciones. En la primera señalaremos los orígenes del término "talento" y su revalorización en el contexto empresarial contemporáneo, para pasar a continuación a repasar uno de los conceptos que ha hecho más fortuna en las últimas dos décadas, el de la denominada marca personal (personal branding), discutiendo sus características e impacto. En la siguiente sección ligaremos talento y marca personal con la emergencia de un nuevo ethos, una personalidad específica neoliberal, propia de este periodo histórico. Finalmente, la discusión final que concluye el artículo se dedicará a reflexionar sobre las implicaciones de esta insistencia en la personalidad adecuada por parte de los nuevos discursos empresariales, apuntando a los desafíos que conlleva para la construcción de una sociedad equilibrada e igualitaria.

\section{Abriendo la caja negra del talento: la razón de un concepto estrictamente empresarial}

Por todas partes nos encontramos con el revalorizado término de "talento" en los discursos hegemónicos de la posmodernidad neoliberal. Este funciona como elemento central en las últimas representaciones de la sociedad del espectáculo, talent shows o programas de supuesto entretenimiento donde se "descubren talentos" o se fabrican en pocas semanas. La moderna pedagogía, en su encuentro con la psicología cognitiva, lo eleva hasta sus últimos niveles en sus pesquisas por encontrar una fórmula de inteligencia práctica. Hasta los diseños de las instituciones educativas de nivel superior se afanan en atraer talentos o evitar la fuga de ellos, aludiendo a una esponjosa excelencia vinculada, finalmente, no solo al esfuerzo sino a un talento especial. No obstante, es desde luego en el mundo de la literatura empresarial, y más exactamente en la autoayuda empresarial, donde el volumen de 
este despliegue de la importancia del talento roza el infinito, adquiriendo polisemias casi barrocas. Ello hace de la actual teoría del management una suerte de retórica liberadora del individuo, que no deja de ser la reencarnación del gastado individualismo metodológico y ontológico liberal de siempre, envuelto ahora en un flamante traje tecnológico (Alonso y Fernández Rodríguez, 2018 y 2019).

La etimología de "talento" nos lleva al talentum latino, que no dejaba de ser una moneda de especial valor en Roma, frente a las monedas genéricas de cobre. Ya tenía su historia en la Grecia antigua, donde tomaba su nombre de tálanton, el plato o el peso de la balanza. Este valor o peso especial fue derivando en su uso hacia las características personales, como por ejemplo una especial capacidad artística, intelectual o intelectiva de los individuos y donde, con la misma etimología, aparece la palabra "talante" para referirse al humor o disposición de ánimo que tiene una persona en concreto. En buena medida, la literatura de consejos empresariales que ensalza tanto el talento está tratando férreamente de forjar un talante, una predisposición positiva y una adhesión absoluta a los valores individualistas, competitivos y flexibilizadores de las nuevas estructuras empresariales en red. Apelar al talento es introducir los discursos de la gestión de empresa en un universo semántico que lo acercan al mundo artístico, en la creatividad individual, la espontaneidad y la capacidad de llevar su proyecto vital hacia delante. Este relato está en el centro de ese nuevo espíritu del capitalismo que analizaban ya Boltanski y Chiapello (2002) en su clásica obra, en la que encontraban en la crítica artística, la presentación de proyectos y las alabanzas de la liberación personal el argumentario básico de la pragmática recurrente para la justificación de todas las decisiones empresariales posfordistas. Desaparecen así las jerarquías, los marcos disciplinarios, las normas y los derechos, el rutinario devenir de conflictos cotidianos en las organizaciones — que habían sido el centro de las escuelas clásicas de gerencia- y aparece el ilusorio mundo en el que todo el mundo tiene talento y solo tiene que atreverse a aprovecharlo sin caer en la desidia, la inactividad, la falta de iniciativa o la acomodación a la seguridad gris de la burocracia. El concepto de talento incrustado en la corriente de los escritos populares de la gerencia y la investigación comercial no puede ser así más difuso: capacidad de resolver problemas, inteligencia práctica, creatividad pura y dura, intuición, vocación, dotación natural, etc. Todo se mezcla y se amplía para nominar una capacidad que tiene un individuo valioso (en el que leyendo este tipo de literatura no dejemos de autoproyectarnos e identificarnos), que puede ser anulada y perdida si lo dejamos a los pies de las grises burocracias o de las masas (o sus jefes o líderes) mediocres y envidiosas.

El género (sub) literario que desarrollan los gurús empresariales, tan representativo de los discursos de la nueva empresa (Alonso y Fernández Rodríguez, 2013 y 2018) opera más por connotación que por denotación (Barthes, 1990: 75-81). Sus consejos, recetas, relatos de grandes hombres o iniciativas felices no sirven tanto para ser reproducidas mecánicamente y puestas en práctica al instante (lo que sería por otra parte imposible), sino porque evocan y construyen un imaginario de éxito, ascenso, disolución de barreras y triunfo del coraje y el mérito. Se acaban importando algunos de los nuevos tópicos de las neurociencias en alza: inteligencia divergente; inteligencias múltiples, pensamiento metafórico, plasticidad neuronal, pensamiento lateral, etc. (un etcétera que podía abarcar 
páginas) para empaquetar unas cuantas sentencias de sentido común que acaban animando a atreverse a ser uno mismo, a aceptar el riesgo sin miedo o a pensar que el que quiere puede. Los marcos sociales e institucionales del comportamiento desaparecen (o se consideran coercitivos de la creatividad), y aparece el gran relato de la autopromoción o del interés empresarial por generar o arrebatar a los competidores seres especiales dotados de dones auténticos. Con el concepto de talento la literatura empresarial ${ }^{3}$ ha desarrollado, de este modo, otro espacio en el que se despliega una guerra de todos contra todos, un mundo hobbesiano en que los individuos tratan de defender su talento frente a otros que entran en la lucha, y en que las empresas se arrebatan unas a otras el talento disponible. Cuando la tierra era el bien más preciado, los poderes se disputaban el territorio físico, pero ahora - dice Tom Peters (2002) usando un tópico empresarial estadounidense - la lucha más encarnizada de todos los grupos, poderes, organizaciones y corporaciones es precisamente por la captación de talento.

El talento, según el mítico Tom Peters (íbid.), se convierte, así, en la variable de la excelencia competitiva esencial para gestionar "en un mundo sin reglas". Identificar y mantener el talento es el centro de la gestión actual de los recursos humanos — según Peters - y entre sus "mandamientos" para la nueva gerencia está en lugar principal el de reconocer a la gente con carisma o la gente que "la gente adora", y por ende, abrirle paso a los jóvenes, a los mejores, a las mujeres, a los bichos raros, a la diversidad y la diferencia. El líder lo es porque tiene, desarrolla y promueve talento, y la forma como este talento es exhibida y es reconocida se convierte en la denominada marca personal. Esta marca personal, cuya implicación, por seguir a Peters (1997) de nuevo, es que la marca eres tú, pasa a ser la variable discriminante absoluta en el mercado de trabajo actual y generadora de las trayectorias profesionales más eficaces, a la que dedicaremos la siguiente sección.

\section{Marca personal: venderse a sí mismo}

Todo el ciclo neoliberal ha venido centrando su labor ideológica en la disolución de la figura tradicional del trabajador asalariado por sus potencialidades políticas para desarrollar una identificación horizontal —una consciencia colectivapotenciando como alternativa uno de los constructos más sintomáticos y más conocidos del discurso dominante actual, el del empresario de sí mismo (Foucault, 2009; Laval y Dardot, 2013; Bröckling, 2016; Barrère, 2018). Esta labor de zapa ideológica ha venido afinándose y complejizándose hasta completar el mito de un sujeto total y exclusivamente entregado a los incentivos de cálculo derivados del mercado, o mejor dicho, de todos los mercados posibles. La colonización mercantil de la personalidad se hace ahora en uno de sus aspectos más importantes, en la misma constitución de su identidad expresada, en la presentación del self, en la exposición y puesta en escena del yo ante los demás en todos los aspectos de la vida cotidiana (Goffman, 1994). La promoción personal y el deseo de realzar una

A lo largo de este artículo, vamos a hacer referencia a cómo aparecen conceptos como talento y personal branding en un conjunto de obras asociadas a la literatura gerencial, cuya selección ha venido determinada por su relevancia a la hora de tratar estos tópicos concretos, y su inclusión en repositorios de bibliotecas universitarias españolas. 
imagen social ante los demás han sido representados, pese a su énfasis en la agencia personal, como un elemento característico de la gubernamentalidad neoliberal (Rose, 1999) y un elemento central del imaginario cultural neoliberal (Hall, 2011). La marca personal llegaba, así, como concepto para expandir y fortalecer el relato de sueño neoliberal de un ser humano solo y exclusivamente socializado en los valores del intercambio mercantil. De acuerdo con Peters (1987), todos tenemos una marca personal, pero al mismo tiempo no todo el mundo ha visto la oportunidad que esta representa como herramienta estratégica en nuestro desarrollo profesional. Todo el alegato sobre la marca personal modula, como código prescriptivo, uno de los aspectos fundamentales de constitución de la personalidad contemporánea: la forma de crear la imagen de uno mismo que se transmite a los otros. Imagen que aquí trata de unificar lo profesional y lo íntimo, de tal forma que el concepto más genuino que caracteriza a la mercancía, la marca comercial, se convierte en el principal identificador de la persona. La modernidad humanista había consagrado un ideal de personalidad basado en la autenticidad y la reflexividad del conócete a ti mismo, donde precisamente la esfera mercantil no podía penetrar, teóricamente claro, en la rectitud y altitud de sus actos (Ehrenreich, 2018: 203). La posmodernidad neoliberal, en cambio, instituye la marca personal como forma correcta de formación del carácter humano en la forma del véndete a ti mismo sin complejos para lograr con ello el éxito íntimo y profesional que ansías.

Esta transición indica que hay un profundo cambio en la constitución de la hegemonía política y, asociado a ello, una sensible variación en la programación ideológica de los modos legítimos de expresión de la identidad personal. En los treinta años gloriosos de la segunda posguerra mundial, el capitalismo era organizado, fordista y fuertemente institucionalizado sobre la negociación colectiva del Estado del bienestar, así como sus formas jurídicas derivadas, algunas de las cuales, en progresivo declive y reformulación, por lo menos parcialmente, todavía llegan hasta nuestros días. Desde entonces, lo que se ha instalado y está hoy en auge es un proyecto neoliberal de capitalismo total, líquido, socialmente desregulado y precarizado. Si hemos asistido, en la esfera de las organizaciones, a una metamorfosis fundamental desde un paradigma de las cadenas a otro de redes (Fernández Rodríguez, 2008), lo mismo podemos decir del individuo, que pasa a asumir un nuevo rol en el espacio del trabajo y la vida. El "hombre organización" típico del fordismo keynesiano estaba centrado en códigos externos de control del comportamiento, en un entorno bien delimitado dentro del contorno corporativo empresarial, con pactos sociales, horarios normalizados, incentivos acordados, carreras profesionales largas y previsibles, derechos laborales, etc. Con el neoliberalismo, hemos venido asistiendo a la formación de un proyecto de sujeto fuertemente individualizado en el que el consumo marca fundamentalmente su identidad social, desaparece cualquier referencia colectiva con respecto al trabajo y sus códigos de conducta se conforman de una manera interna - de autocontrol-, esto es, por asimilación total e ideal de la persona, pensada como soberana y absolutamente completa, de los valores totales del ideario liberal-mercantil.

En esta reconfiguración mercantil de la personalidad, se aspira a la entrega total del individuo a los valores, misiones e intereses de las empresas, con independencia de su estatus en la organización. La ideología del empresarialismo total persigue, ante todo, conseguir el "compromiso", esto es, la fidelidad absoluta del asalariado ya 
no solo en las ocho horas reguladas de la jornada laboral canónica, como antaño, sino la entrega de su vida entera misma, desde todo su tiempo sin restricciones hasta sus afectos y emociones, desde su disponibilidad a su felicidad (Cabanas e Illouz, 2019). Como han señalado algunos de los estudios más perspicaces en la sociología organizacional, la cultura de empresa se ha terminado convirtiendo en la cultura en sentido estricto, obligando a una reconstrucción de las identidades de los individuos, en la que ciertas cualidades empresariales son elevadas a la categoría de virtudes de la persona (ver Miller y Rose, 1990; du Gay, 2012 y 2019). El yo posmoderno de los asalariados más cualificados, su yo público, se construirá en torno al eclecticismo y la reinvención, tal y como hacen las marcas en tiempos de cambios continuos, pero siempre con el dinero y la ganancia como único elemento sólido en la existencia humana (Berger, 2011). La comunión ideológica con los objetivos empresariales se convierte en condición de supervivencia en el entorno del capitalismo de redes, tanto para aquellos que ocupan una posición supraordinada y promocional, y que ejercen un individualismo proactivo y con exceso de capacidades, como para los que se incrustan en posiciones subordinadas y precarizadas (Candil, 2020; Landa, Blázquez y Castro, 2019). Estos últimos, de hecho, se ven obligados a sobrevivir con un individualismo defensivo, precarizado y buscavidas, con déficit de oportunidades solventes (Castel, 2010; Alonso, 2007; Muñoz-Rodríguez y Santos Ortega, 2017).

En este marco ideológico, el consumo resplandece como el principal motor de los estilos de vida actuales, ocultando en gran medida los lugares o el origen laboral de la posición social; del orgullo del trabajador del relato obrero clásico o del puritanismo productivista típico de las clases medias funcionales corporativas, hemos pasado a la exhibición de mercancías y la exposición de objetos con marca como forma de representación social principal del estatus y el atractivo social de las personas. La marca se ha convertido en la era del consumo en el identificador social universal (Alonso, 2005), y así, de la marca comercial restringida al mundo de los objetos - donde la marca tiene hoy el dominio semántico sobre el propio producto (Baudrillard, 2011)—, hemos pasado a hablar de la marca país, de la marca ciudad, de la marca profesional o de la marca personal (por solo citar unas cuantas). Con ello se expresa algo que ya queda como naturalización incontrovertible de nuestro imaginario social contemporáneo, en el que el significante ha absorbido al significado, donde la imagen es más importante que el contenido, y donde el valor reputacional se deriva más del lugar que ocupa un producto/marca en el sistema general de signos — que es la actual cultura global de consumo - que de las prestaciones reales y tangibles de la mercancía. El valor de signo controla y determina el valor de cambio y el valor de uso no solo de los objetos, sino ahora, también, de las personas (Baudrillard, 1974 y 1978a).

Este proceso de preponderancia del valor de signo se ha traspasado a la literatura de la gestión empresarial y, en consonancia con ello, se ha puesto en primera línea del vocabulario del managerialismo popular ese pseudoconcepto de marca personal. En realidad, la literatura desplegada bajo este rótulo es tan heterogénea, diversa, ambigua y confusa como en este género estamos acostumbrados a encontrarnos, siendo una mezcla de gerencialismo popular, autoayuda empresarial y superación personal. Aquí, otra vez, el concepto de marca personal sirve para ocultar el lado estructural de las relaciones laborales, desplegando un individualismo mágico —en el sentido ritual/antropológico del 
término- y reduciendo el conflictivo mundo de la gestión de los recursos humanos a una simplista visión de estrategias de marketing personal, especular y autopromocional.

El impacto de la marca personal ha sido extraordinario a nivel discursivo, con la proliferación de textos empresariales centrados en exponer los beneficios del personal branding. No es de extrañar que, de nuevo, haya sido Tom Peters, uno de los más reputados gurús de la gestión, el encargado de flotar - y fletar- un significante con tanto éxito en el campo del managerialismo popular como el de la marca personal, desde su artículo de 1997, "The Brand Called You", luego recompuesto en otro de los libros que volvieron a entrar en la tan buscada categoría en este sector de best-seller de la gestión (Peters, 2000). El argumento básico, como era de prever, es extremadamente simple $-\mathrm{y}$ ya lo había perfilado otro famoso gurú, Daniel Pink, en una serie de artículos de los años noventa luego también convertidos en libro (Pink, 2001), cuando trataba de convertir todo el mundo salarial norteamericano en un mercado de "agentes libres"- : el mercado laboral selecciona las señales positivas, reconoce a las personas que presentan una apariencia de notoriedad, consistentes y atractivas para los usos empresariales del momento. Es responsabilidad del individuo que quiere ser reconocido en el mercado de trabajo componer su imagen como una marca positiva, notoria, consistente y adaptada a los perfiles incluso psicológicos y emocionales que demanda el mundo empresarial en esos momentos.

$Y$ es que en un mercado del trabajo cualificado crecientemente saturado, parece hacerse cada vez más imprescindible sobresalir entre esas masas de trabajadores formados, y a ello nos puede ayudar la marca personal (McNally y Speak, 2002), presentada en multitud de libros y artículos tanto académicos como divulgativos como una auténtica revolución para las carreras de la gente (Arruda y Dixson, 2007). Ello nos va a permitir transformarnos en una marca (Montoya y Vandehey, 2003) susceptible de ofrecer, a corto, medio y largo plazo, grandes dividendos (Kang, 2013). La necesidad de aparecer, ante los ojos del mercado, como alguien único, valioso e insustituible nos obliga a trabajar en nuestras singularidades, en nuestra identidad, buscando diferenciarnos de los demás (VV.AA., 2011). Sin embargo, esta diferenciación no hace referencia a ningún elemento bohemio, sino que debe generar buena imagen (lo que implica tener un especial cuidado de sí mismo) y sobre todo confianza en aquellos que pueden contratarnos, comprarnos, o hacer negocios con nosotros. Autores como Rein et al. (2006) han considerado que, en la actualidad, los individuos tienen la oportunidad de tomar prestadas técnicas propias del mundo del marketing y aplicarlas a su vida personal, con el objetivo de aspirar a una mayor visibilidad: cuando alguien es exitoso en la construcción de dicha marca, esa persona tiene la posibilidad de ser más conocida y más capacidad a largo plazo de estar en una buena posición en el mercado. La cuestión clave aquí va a ser la de posicionarse estratégicamente, lo que supone tanto el posicionamiento de uno como el de su carrera profesional (Ries y Trout, 2001), del mismo modo que hacen las empresas. Y como estas, nuestra marca personal debe cuidarse al máximo, en especial en lo que se refiere a su reputación e imagen ${ }^{4}$.

Incluso los propios empleadores van a obsesionarse con su marca corporativa y su capacidad de atraer y retener empleados, lo que les obliga a modificar sus políticas de recursos humanos, en las que las nuevas estrategias serán las de la gestión del compromiso, de las personas y del talento, en línea con el discurso de la marca personal (ver, como ejemplo, Aguado y Jiménez, 2017). 
Naturalmente, dentro de ese importante mercado que es el de la literatura gerencial dirigida específicamente a mujeres (Medina-Vicent, 2019), no podían dejar de aparecer textos dirigidos al desarrollo de una marca personal, destacando trabajos como el de Roffer (2000), cuyo objetivo es el de proporcionar consejos a las profesionales acerca de cómo afianzar esa imagen profesional.

Estas ideas han tenido un impacto notable en el mundo del trabajo, en especial en algunas profesiones de la denominada economía del conocimiento. Las clases creativas que describe Richard Florida (2010) son las que más se ven expuestas a la necesidad de crear su propia marca en un contexto progresivamente mercantilizado $\mathrm{y}$, a la vez, hambriento de perfiles especializados. En las nuevas organizaciones expresivas se genera un juego de identidades novedoso, en el que se establecen relaciones complejas entre la marca corporativa y la personal (Schultz, Hatch y Larsen, 2000). De este modo, se han realizado estudios sobre el impacto que la marca personal ha tenido en sectores como los ejecutivos de empresa (Nolan, 2015), médicos (Luca, Ioan y Sasu, 2015), periodistas (Brems et al., 2017), artistas y diseñadores de arte (Kucharska y Mikolajczak, 2018), o incluso comerciales y vendedores (Amoako y Okpattah, 2018), y no digamos ya a los académicos que, pese a permanecer en buena medida empleados en el sector público, han terminado asumiendo los presupuestos del personal branding en un mercado de la atención y la cuantificación de las métricas. En todos los casos el discurso de la marca personal parece haber penetrado con mucha fuerza. De hecho, incluso algunos trabajadores de ciertas profesiones cualificadas pero precarizadas lo asumen (López Salas y Urraco Solanilla, 2018), si bien con algunos matices (Vallas y Christin, 2018). Se dice además que los jóvenes profesionales, de hecho, se beneficiarían notablemente de una construcción rápida de su marca personal, ya que no solamente les va a permitir conocerse mejor (entendiendo esto desde una perspectiva en la que la consideración básica es la de concebir la personalidad del individuo como un conjunto de debilidades y potencialidades), sino planificar sus estrategias de desarrollo personal desde su incorporación al mercado de trabajo. De hecho, según Ilies (2018), lo ideal sería que la marca personal se trabajase ya desde los estudios, lo que les permitiría tener una mejor orientación profesional y establecer redes desde el principio de su trayectoria laboral.

$\mathrm{La}$ intersección entre identidad y reputación tiene, en resumen, efectos indudables en la posición del trabajador en la organización. Además, la marca personal termina trascendiendo la esfera profesional, en un proceso de disolución de las barreras entre trabajo y vida característico de la era neoliberal. Progresivamente, la presencia en las redes sociales termina conduciendo a que lo personal también devenga en profesional, con una comunicación personal controlada y adaptada a los requerimientos de la corporación (Nolan, 2015), dentro de un régimen de economía de la atención (Goodwin et al., 2016). Así, espacios como Twitter, que vinculan una cuenta de la red social a un nombre concreto, permiten a los profesionales construir de forma activa una marca que les permite resituarse como no solamente expertos profesionales, sino además les ofrece una oportunidad de atraer y canalizar una audiencia hacia su trabajo y el de la corporación para la que trabajan (Brems et al., 2017). El yo, la identidad personal, se convierte así en una verdadera herramienta de trabajo y las redes sociales se 
convierten en esa extensión del yo, hasta el punto de poder hablar de un Yo 2.0 (Schawbel, 2009).

Evidentemente, en este desplazamiento del significante al significado en el mundo laboral, el lugar de las mal llamadas redes sociales y la forma de la presentación de la persona en internet se intensifica y magnifica, hasta hacerse prácticamente el argumento principal, por no decir único, de conformación de las estrategias actuales para llevar a cabo un proyecto de creación de marca personal. Las redes sociales son el paradigma de la profundidad y consistencia comunicativa que se busca en estas maniobras; son contactos esporádicos y superficiales, que forman una presencia más cercana al simulacro (Baudrillard, 1978b) que a una recopilación o catálogo de méritos tangibles verificados. El significante "red" aparece por todas partes - ha sustituido de hecho a la idea de pirámide organizacional característica del gerencialismo clásico - porque introduce una dimensión etérea, sin sujetos fuertes, sin límites fijos, de supuesto fácil acceso y sin poderes excesivamente visibles. Si comparamos la obsesión actual, en todo este tipo de modas empresariales, por el networking — banalización instrumental de las relaciones personales para fomentar el negocio - y lo comparamos con los paradigmas clásicos de la escuela de las relaciones humanas de Elton Mayo y sus trabajos sobre clima laboral y grupos formales e informales en el entorno productivo, nos daremos cuenta hasta qué punto la idea del medio social y sus determinaciones se ha ido minimizando e individualizando en la literatura gerencial actual hasta convertirla en un puro accesorio estético.

De esta manera, frente a la idea de cualificación, conocimiento, oficio, predisposición o incluso fidelidad, valorados como elementos determinantes de la puesta en valor de la oferta laboral en el discurso clásico de los recursos humanos - o sea, los contenidos reales de esta oferta-, ahora en el discurso de la marca personal o la marca profesional es la imagen proyectada lo que cuenta. Se trata de la construcción, supuestamente consciente, de un tipo de personalidad que pueda seducir en todas las dimensiones de la vida — pública y privada-, pero fundamentalmente en aquellos aspectos que se convierten en marcadores eficaces para el éxito profesional. Todo estos "efectos halo" irradiados por la personalidad (seguridad, competencia, accesibilidad, rigor, etc.) se pueden entrenar gracias a las enseñanzas de los gurús, de mentores, del coaching, de los libros de autoayuda empresarial o de los saberes que nos ha trasmitido la nueva inteligencia emocional. $\mathrm{Y}$ todo esto parece que sirve de forma infalible para vendernos mejor en el mercado de trabajo. Con ello, parece que está todo dicho sobre cómo se lleva a cabo la selección de personal en las organizaciones complejas y, en general, en la sociedad actual: siempre se elige a quien mejor marca personal tiene y presenta, lo mismo que en el mercado de productos escogemos la marca más atractiva.

La idea de marca personal no es otra cosa que una teoría de la señalización en los mercados profesionales. En los textos gerenciales especializados en el tema, lo que se viene a decir a ese posible lector de este tipo de literatura es que tiene que asociar, como es obvio, el mayor número de atributos positivos posibles a su persona - convertida ya en marca comercial-, recalcando el valor de la imagen proyectada en todos los espacios de la vida (porque hasta los que parecen más lejanos de la estricta vida laboral tienen repercusión en el éxito profesional). Y, finalmente, el individuo que está perfilando y cuidando su marca debe conseguir 
ser sobresaliente, relevante y diferente, en contraste con la mediocridad generalizada o el anonimato que impone una sociedad que produce enormes cantidades de titulados, fuertemente normalizados en sus competencias y habilidades, y escasamente distinguibles a ojos de empresas ávidas de talento.

\section{La personalidad neoliberal}

A principios de los años cincuenta del siglo pasado, Theodor Adorno y su equipo buscaban un patrón de personalidad autoritaria, que había sido el caldo de cultivo psicológico para el advenimiento de los fascismos, pero que, en general, se demostraba básico para el peligroso éxito y la enorme recepción de las ideas antidemocráticas de su época, que llegaron a tener un seguimiento importantísimo en todos los países occidentales, incluso en aquellos finalmente decantados por la defensa de la democracia (Adorno, 2009). Independientemente de las polémicas sobre la verosimilitud y plausibilidad de los métodos y hasta de las conclusiones de los estudios de Adorno, sí que es interesante hacer notar la importancia de su habilidad para encontrar pautas de comportamiento político que tienden a estar encajadas en modelos de personalidad, producidos por distintos factores: sobresocialización, adhesión al superyó paterno y conversión de la inseguridad personal en agresividad hacia el otro, visto como diferente o extraño. En la actualidad, parece que la personalidad autoritaria de Adorno se ha ido transmutando y transfigurando, para reaparecer como proyecto ideológico en una personalidad neoliberal, donde toda la socialización, la formación de estereotipos y la adhesión a los valores fundantes se hace ajustándose, en este caso, al estrecho repertorio de valores derivado de lo estrictamente mercantil.

Conceptos como talento o marca personal, usados en este contexto, están en esa línea de reducir la persona a la personalidad mercantil, donde hasta las relaciones intersubjetivas se configuran semánticamente como un proceso de oferta, demanda y precios (reales, figurados o simbólicos). La empresa total (por seguir el término de Curcio, 2005) se impone, por tanto, como régimen general de enunciación. En este, como dice el sociólogo italiano Vanni Codeluppi (2000: 35-41), la puesta en escena de las personas en el espacio público ya no está hecha en función de su fuerza de trabajo como valor de uso de sus habilidades y cualificaciones — - según la teoría de la división del trabajo del industrialismo clásico-, o desde al valor de cambio — según la posición crítica del marxismo igualmente clásico-. Más bien, esta puesta en escena se hace como valor signo, espacio donde se reproduce el espectáculo de la mercancía (capacidad de consumo, seducción, soporte de marcas, atracción deseante, etc.). La reflexividad, cualidad del individuo en la modernidad tardía (Giddens, 2000), será completamente mercantilizada, con su correspondiente apropiación por parte de algunos de los actores sociales y la exclusión de otros, generando nuevas formas de capital cultural y creando nuevas desigualdades (Wee y Brooks, 2010). La marca personal, así, puede entenderse asimismo como un proceso susceptible de ser adquirido, consumido e internalizado por los agentes del mercado, y que les permite convertirse en ese Yo que va a ser visto.

La obsesión por esta exposición mercantil del yo ha creado figuras bien conocidas en el mundo de la nueva economía, como la del yo cuantificado, 
movimiento proclamado desde la revista Wired y otros focos de la economía digital actual (ver Fuller, 2015), y que impulsa a la personas a medirse a sí mismas en todas sus dimensiones posibles y publicar e intercambiar permanentemente los resultados de sus mediciones como datos en todos los foros digitales conocidos (self-tracking), tanto profesionales como de ocio o amistad. Una cultura del rendimiento y a la vez de la perfomance (Ehrenberg, 2009) se instala en cada minuto, se miden seguidores, "me gustan" o replicaciones de mensaje, pero también las horas dedicadas a comer, a correr (siendo el deporte y el cuidado de la salud uno de los principales impulsores del movimiento del Quantified Self), a hacer bricolaje o a tocar un instrumento, etc. Todas ellas se exponen en la aplicación informática que sea (las aplicaciones son lógicamente otro negocio complementario en crecimiento exponencial) en tiempo real, para mostrar así una capacidad de acción, los logros personales, la proactividad y el éxito particular en todas sus variedades. La marca personal ya se puede ofrecer con datos y puntuaciones, y el no registro de nuestras actividades o su no validación por un sistema de normalización y difusión informático se sale de las prescripciones dominantes y te relega a la invisibilidad y la exclusión, equivalente, en ciertos contextos profesionales y sociales, a la muerte social (Friedrich, 2018: 65-66).

En todo este despliegue ideológico de la cultura neoliberal el yo se ha cosificado y convertido en mercancía — según analiza Barbara Ehrenreich (2018: 207) - que requiere un mantenimiento y puesta al día permanente para conservar una marca conocida y distinguible. La fama y los famosos parecen el ejemplo perfecto de marcas triunfantes, seguras y reconocibles al primer vistazo, y este es un modelo que se difunde como aspiración general en el capitalismo tardío. El mandato se vuelve otra vez paradójico: todo el mundo tiene que diferenciarse haciendo las mismas cosas, diciendo que tiene una personalidad positiva, creativa, adaptable emprendedora, etc. Por supuesto esto implica una fortaleza de carácter que se consigue a base de autoestima, confianza en sí mismo, coraje y fuerza de voluntad, y cuando aparecen dudas, miedos o zonas oscuras (lo que seguramente indica un carácter apocado, romántico o melancólico, poco compatible con el nuevo espíritu del capitalismo), siempre se puede acudir a la meditación, al mindfulness, al yoga, la autoayuda o las psicoterapias varias y, en el caso de profesionales, al recurso de un coach.

Como muy bien ha estudiado la socióloga franco-israelí Eva Illouz, la programación de las emociones es una aspiración capitalista que se va profundizando en cuanto más avanza y se expande la ideología de mercado, de tal forma que la propia estandarización de los comportamientos emocionales es tomada como un factor fundamental para predecir y controlar la productividad económica, así como para clasificar y distribuir a los individuos en los sistemas funcionales corporativos (Illouz, 2014: 57-59). De hecho, en la nueva economía digital y financiera se da por sentado que lo que más obstaculiza las condiciones de alto crecimiento económico no son las constricciones técnicas, la escasez de recursos o las determinaciones naturales, sino las propias creencias y las convenciones generalmente aceptadas (Orléan, 1999: 85). Remedios Zafra (2017: 106) lo expone cuando describe las fórmulas de autoengaño, de ese "querer creer" con el que se fabrican diariamente los imaginarios contemporáneos y que ya no solo son formas de evasión, ficción y entretenimiento, sino formas de ajuste suave 
a la disciplina productiva. En el ideario de las doctrinas empresariales posmodernas, a cambio de inseguridad y precarización se ofrece una libertad y creatividad en las que hay que "querer creer". Se exige en la maniobra un compromiso que ya no es simplemente el cumplimiento de una norma contractual o de la relación salarial misma, sino un auténtico entusiasmo, no tomado este concepto, a la forma del ideal clásico, como forma de exaltación derivada de una pasión intelectual y creadora, sino prescrito en su versión de la nueva economía digital, esto es, como "apariencia alterada que alimenta la maquinaria y la velocidad productivas en el marco capitalista" (Zafra 2017: 16). En este entorno posfordista parece fundamental superar la convención industrialista, sancionada por el contrario de trabajo y llevada a sus últimos efectos por el taylorismo y la organización científica del trabajo, de separación institucional entre el trabajo y la fuerza de trabajo, o entre la prestación laboral y el cuerpo del trabajador. La marca personal, el entusiasmo, la flexibilidad, la disponibilidad, la activación, el pensamiento positivo y demás voces del diccionario del managerialismo actual son buena prueba de ese intento de ampliar y hasta completar o saturar todo el espacio y el tiempo de vida de las poblaciones con la obligación de producir valores (materiales y simbólicos) que refuercen el poder mercantil (Zarifian, 2001).

Un conjunto de investigadores italianos ha denominado a este proceso la construcción de una gubernamentalidad por imprinting o imprimación (ampliando el modelo canónico de subordinación/subsunción del trabajo en el capital analizado por el marxismo clásico), es decir, por un modo de producción de subjetividades que consagra, a priori, la legitimación y validación de una lógica de mercado total: "He aquí la doble imposición del imperativo categórico del capitalismo contemporáneo (1) sé lo que quieras, lleva a la práctica tu autonomía, bajo la condición de que (2) la resultante de la acción sea traducible en la axiomática del capital y sus métricas convencionales en constante mutación" (Chicchi, Leonardi y Lucarelli, 2018: 62). Esta imprimación o impresión se vuelve a plantear en forma paradójica y a la vez pragmática. Es un tipo de socialización basada en la máxima individualización o, de otra manera, es un dispositivo de gobierno que organiza la producción y control social incitando a la máxima autonomía subjetiva, discursivamente presentada como la consagración absoluta de la libertad de elegir. Se trata, en definitiva, de una suerte de creación, construcción de "personas", tal como lo definen algunos autores como Hacking (1986) o du Gay (2019).

No era de extrañar que en toda esta mixtura de pseudoconceptos, el tema de la marca personal y profesional se acabara mezclando con el recurso a lo emocional tan en boga en estos últimos años, y que caracteriza algunos de los desarrollos más significativos de la colonización de la vida íntima por parte del mercado (Hochschild, 2011; Illouz, 2019). Por lo tanto, en el perfil a entregar - y a exponer en las redes sociales y en todos los escaparates de internet, ante todo - no se puede hacer un seco comentario de las cualificaciones o competencias del individuo en cuestión, sino abrir en su lugar un amplio repertorio de buenos sentimientos y virtudes afectivas en una demostración de carácter amigable, pero enérgico. Lo mismo ocurre con la recurrente apelación a la creatividad, otro significante al uso y abuso para completar la marca personal (Alonso y Fernández Rodríguez, 2018; Mould, 2018). La personalidad ofertada tiene que ser así flexible, ocurrente, con capacidad de resolución de problemas, sensible, curiosa, inquieta, etc. (un etcétera 
que podría ocupar páginas), pero que básicamente conduce a la vía imaginaria a alcanzar una mentalidad abierta a la inestabilidad, el emprendimiento, la autovigilancia y el rechazo a la seguridad burocrática (siempre connotada como la expresión de la maldad misma). O, dicho de una manera más directa, una mentalidad predispuesta a la mayor disponibilidad posible, sin la mínima resistencia a cualquiera de las exigencias del modo de regulación posfordista.

La socióloga Arlie Russell Hochschild, continuadora de la mejor sociología crítica norteamericana de C. W. Mills o Erving Goffman (Oliveira-Martíns, 2018: 38-45), ha ido entregando una monumental obra sobre la gestión de las emociones y la estrategia de los individuos para la protección del yo, como un yo sensible que se siente acosado por la despersonalización, la insignificancia o el aislamiento. Para Hochschild, la empresa se sirve de la capacidad personal de gestionar las emociones tratando de intervenir en la construcción de las reglas de los sentimientos con las que se regula el trabajo emocional. Controlando así los sistemas de interacción y socialización, el sistema de afectividades se pone al servicio de los propósitos empresariales. Siempre hay la posibilidad de que la adhesión emocional a esta mercantilización sea incompleta, parcial o puramente superficial y adaptativa; pero precisamente toda la literatura managerial y el discurso de la cultura de consumo dominante está hecho para que la adhesión sea profunda, completa y pasional "cuando los gestos profundos de intercambio ingresan en el sector mercantil y se compran y venden como aspectos de la capacidad laboral, los sentimientos se mercantilizan" (Hochschild, 2011: 151). A fin de cuentas, en el contexto del capitalismo de redes y su mercantilización de todo, la propia reflexividad de los individuos es susceptible de mercantilizarse, e incluso se generará el correspondiente mercado de expertos que proporcionará ese conocimiento necesario para construir un marketing de la identidad (Wee y Brooks, 2010).

\section{Conclusión: La justificación de la desigualdad o el racismo de la inteligencia}

Toda la narrativa empresarial sobre el talento - $-\mathrm{y}$ sus derivados en las administraciones públicas o en la gestión educativa posmoderna- está presidida por una abrumadora y casi agresiva descontextualización sociohistórica. En este discurso, el talento se convierte en una especie de sustancia natural, que se puede producir en cualquier tiempo y lugar, que aparece como un don de creatividad e imaginación inesperadamente y que solo se asocia a individuos especiales que pueden surgir libre y azarosamente. El talento, así, primero es, por definición, indefinible — todo puede ser talento-, pero además, cuando se trata de definir, se ocultan sistemáticamente los orígenes sociales e institucionales de tanto lo que el sistema mercantil valora subjetivamente como talento - esto es, la máxima adaptación ideológica al sistema de valores promocionales de la competitividad interpersonal-, como de las condiciones objetivas y desiguales en las que se realizan los procesos de formación, educación y adquisición de méritos que terminan siendo valorados como talentos. El talento -aunque los gurús empresariales no tengan especial interés en observarlo- se genera, y se valoriza, 
en procesos sociales, y arrastra tras de sí las condiciones estructurales desiguales en las que se produce esa realidad social.

Hablar universalmente de talento deja sin sentido de manera implícita el uso de términos tradicionales en el mundo de la empresa como oficio o cualificación. Ambos nos remitían a un saber hacer social, a una capacidad de reflexión y control del trabajador sobre su propio proceso productivo, conceptos ligados ambos a categorías formativas socioprofesionales a las que se atribuye, institucionalmente, un lugar en la división social del trabajo, y que históricamente han sido objeto de conflicto y negoción colectiva en los sistemas modernos de relaciones laborales (Braverman, 1974). Desaparecen, por tanto, los temas centrales en la organización de empresas como disciplina disciplinadora del trabajo en las corporaciones. En los nuevos discursos gerenciales, al apelar al talento, los recursos humanos quedan reducidos a la responsabilidad estricta del individuo, que debe activarse y publicitarse, renunciar a derechos o adaptarse a las demandas organizativas que se supone lo están dirigiendo por el camino del éxito, y con ello se convertirá en un objeto de valor máximo por el que lucharan las empresas. El requerimiento al talento circunscribe, definitivamente, su sentido a un fuerte proceso de individualización y al ocultamiento de los factores sociales e institucionales que determinan las posiciones de los individuos en las escalas valorativas de la gerencia de recursos humanos en la empresa, contribuyendo con ello a crear la obligación de abordar la autoconstrucción de una imagen del yo (self) como identificación perfecta con el clásico egoísmo del interés liberal, ahora relegitimado bajo el manto del genio, la innovación y la creatividad.

El discurso del talento y la marca personal, tal como es difundido por la literatura managerial en boga, busca así un efecto performativo, esto es, activa un uso del lenguaje que no proporciona ninguna información o declaración susceptible de verdad o falsedad, sino que intenta crear el acto enunciado por el hecho mismo de realizarlo (Austin, 1981). De esta manera nos encontramos ante todo un género de enunciaciones en que el mero hecho de decir y difundir algo produce la acción buscada - como en las promesas, como en las órdenes - y que, en este campo, no es otra cosa que naturalizar, desocializar y aislar al individuo en la organización económica. Es un lenguaje normativo que se disfraza de psicológico, clínico o tecnocientífico, pero que no aspira a otra cosa que a proclamar su autorrealización; siempre jugando a ser un género descriptivo, al faltarle anclaje y relevo - o relación significativa con contextos sociales concretos (Barthes, 1971) - resulta pura y abiertamente prescriptivo.

Por eso al tratar de entrar en los enunciados concretos de esta literatura de la marca personal — como en los libros de autoayuda o de mejora del yo-, aparece siempre su carácter performativo, sus actos de habla no son ni verdaderos ni falsos, sino pseudoproposiciones que lejos de reflejar o constatar hechos sociales aspiran al éxito y efectividad de la propia palabra que enuncian. La enunciación constituye, por sí misma, cierto acto, entendido como transformación de las relaciones entre los interlocutores y los referentes. El discurso performativo - lo sabemos gracias al pensamiento pragmatista (Searle, 1986; Escandell Vidal, 1993) - es capaz de crear representaciones sociales, por cuanto una representación social es tanto un contenido como un proceso que induce formas de conocer al grupo social, lo que significa que no solo es un producto de la práctica colectiva, sino un conjunto de dimensiones 
imaginarias que construye y limita la socialidad misma (Jodelet, 1986). El lenguaje tiene una centralidad total en la nueva empresarialización del mundo, porque como ha estudiado Stuart Hall, es el carácter performativo del lenguaje el que le da su potencialidad de visibilizar y ensalzar partes de lo social y ocultar o trivializar otras y, de esta forma, si por medio de los discursos se crean subjetividades empresariales, tendremos sujetos que se comportarán socialmente como empresarios en todas las dimensiones de su existencia humana, y toda la cultura se reducirá a una cultura empresarial, esto es, a un sistema de mandatos mercantiles (Hall y Merlino, 2011).

El reverso de todo este discurso nos está remitiendo a un subtexto bastante oscuro, pleno de darwinismo social, desprecio por los que no pueden, saben o quieren competir, e implica una naturalización indirecta de la desigualdad derivada de la posesión de habilidades que se presentan ahora como estrictamente personales. Esto supone que en la modelación del yo hay un elemento fuertemente moralizador e individualizador (Skeggs, 2004). Nada se dice de los que no tienen ese supuesto talento, de los que pierden en la competencia, de los que no pueden salir o no son "descubiertos"; pero como implicación convencional (Grice, 1991) de este enunciado se deriva que los talentos no reconocidos son talentos perdidos (o ni siquiera son talentos), puesto que el reconocimiento del talento se realiza por un aparato institucional y utilitario, por un poder establecido (privado o público, educativo o profesional) que reconoce talento para convalidar y aumentar su dominio o su aspiración a dominar. En una palabra, nos encontramos ante una nueva versión de lo que Pierre Bourdieu denominó en su día, el racismo de la inteligencia, una forma sutil de construir un relato de discriminación radical: "Este racismo es propio de una clase dominante cuya reproducción depende, en parte, de la transmisión de capital cultural, capital heredado que tiene la propiedad de ser un capital incorporado y, por tanto, aparentemente natural, innato. El racismo de la inteligencia es lo que utilizan los dominantes con el fin de producir una teodicea de su propio privilegio, como dice Weber, es decir, una justificación del orden social que dominan. Es lo que hace que los dominantes se sientan justificados de existir como dominantes, que se sientan de una esencia superior" (Bourdieu, 2000: 261-262).

Como hemos visto, la exhortación del talento se ha convertido en uno de los ejes centrales de la construcción de la personalidad neoliberal. Pero esta exhortación es discursivamente contradictoria y ambivalente. Encierra además una paradoja pragmática: se celebra la vocación, la libertad creativa y el anticonservadurismo, pero, a la vez, se ensalzan los valores dominantes, el máximo ajuste a los objetivos empresariales y el seguimiento acrítico de los valores mercantiles más convencionales (empezando por el canto de la conversión automática del talento en dinero puro y duro). La capacidad crítica o las prácticas no estrictamente sancionadas por el éxito empresarial se quedan fuera del discurso convencional del talento, y con ello, la idea de libertad creativa es sencillamente una trampa semántica. El doble vínculo (Bateson: 1985; Watzlawick, Beavin y Jackson: 1971), esto es, el mandato que se anula en su propio enunciado queda así explicitado: "Ten talento, se libre, pero haz aquello que te digo", "se creativo, pero limítate a seguir la norma empresarial", en línea con otras paradojas propias de los discursos gerenciales contemporáneos (Fernández Rodríguez, 2007). Aquí, el plano del contenido está directamente enfrentado al plano de la enunciación, y la ambivalencia y la inseguridad en el comportamiento se diseminan por todas las 
partes de este género tan lleno de consejos y admoniciones: nunca se sabe ni cuanto talento, ni qué talento es necesario para triunfar, pero sí se transmite como metamensaje que el fracaso es, seguro, por falta de talento. Como todo doble vínculo, el discurso convencional y mercantil del talento es la vía de entrada para construir una interacción social patológica y específicamente esquizofrénica; hagas lo que hagas, si las cosas no salen bien, la culpa siempre cae del mismo lado: el individuo autoculpabilizado. La individualización, por tanto, siempre parece positiva, pero oculta un carácter profundo considerado como negativo; todo vale para invisibilizar las diferencias entre los grupos sociales y sus influencias y resultados sobre las trayectorias personales.

La perversión y el retorcimiento de un concepto tan histórico y naturalizado como el de talento, o la flotación de un significante tan intelectualmente débil y remercantilizador como el de marca personal se inscriben en todo un movimiento de empresarialización de la vida cotidiana, a partir de la empresarialización de la subjetividad humana misma. Estos discursos aparecen como mandatos, recetas o admoniciones desde la individualidad más absoluta y presuponiendo que la voluntad de cambio personal es suficiente para la mejora, la superación o el éxito. En todo este movimiento de exaltación del yo ha desaparecido la estructura social, las posiciones en la división del trabajo o los constreñimientos debidos a los contextos históricos concretos. El tipo ideal de un ejecutivo, masculino o masculinizado, del mundo de la economía de los servicios, de las clases medias promocionales y con aspiraciones ascendentes se trata de hacer pasar por una especie de ser humano universal que tiene los mismos deseos, herramientas y posibilidades en cualquier lugar del tiempo y el espacio. Pero no sabemos si estos juegos del lenguaje serán suficientes para legitimar y naturalizar la precarización, la brecha salarial de la mujer, la desigualdad creciente en todos los órdenes, los malos empleos y los horarios abusivos, por solo citar algunas de las zonas oscuras del mercado laboral y las relaciones industriales actuales. Parece, en definitiva, que nos encontramos en el centro de una guerra cultural por el sentido y los significados de lo que tiene que ser nuestra vida en el trabajo, más allá del entorno físico y de los límites tradicionales de la empresa. La ofensiva desde la lógica del mercado está muy clara, trata de construir una ideología del rendimiento y de la performatividad de la razón económica que impregne todos los aspectos de nuestra existencia, desde las prácticas más visibles a los sentimientos más íntimos.

\section{Bibliografía}

Adorno, T. W. (2009): "Estudios sobre la personalidad autoritaria", en Escritos sociológicos II, vol. 1, Madrid, Akal.

Aguado, M. y A. Jiménez (2017): Empresas que dejan huella. Employer branding en la sociedad conectada, Madrid, Almuzara.

Alonso, L. E. (2005): La era del consumo, Madrid, Siglo XXI.

Alonso, L. E. (2007): La crisis de la ciudadanía laboral, Barcelona, Anthropos.

Alonso, L. E. y C. J. Fernández Rodríguez (2006): "El imaginario managerial: el discurso de la fluidez en la sociedad económica", Política y Sociedad, 43 (2), pp. 127-151. 
Alonso, L. E. y C. J. Fernández Rodríguez (2013a): Los discursos del presente, Madrid, Siglo XXI.

Alonso, L. E. y C. J. Fernández Rodríguez (2013b): “Los discursos del management: una perspectiva crítica", Lan Harremanak, 28, pp. 42-69.

Alonso, L. E. y C. J. Fernández Rodríguez (2018): Poder y sacrificio: los nuevos discursos de la empresa, Madrid, Siglo XXI.

Alonso, L. E. y C. J. Fernández Rodríguez (2019): “Gerencialismo y ficción, o la ficción del gerencialismo: de Ayn Rand a las novelitas de ejecutivos", Debats. Revista de cultura, poder y sociedad, 133 (1), pp. 77-94.

Amoako, G. K. y B. K. Okpattah (2018): "Unleashing salesforce performance: The impacts of personal branding and technology in an emerging market", Technology in Society, 54, pp. 20-26.

Ampudia de Haro, F. (2019): "Gerencialismo universitario y publicación científica", Debats, 133(1), pp. 47-62.

Arruda, W. y K. Dixson (2007): Career Distinction: Stand Out by Building Your Brand, Hoboken, NJ, Wiley.

Austin, J. L. (1981): Como hacer cosas con palabras, Barcelona, Paidós Ibérica.

Barrère, C. (2018): "Discipliner la consommation”, en Roux, Dominique y Giaquel, Yohan (eds.), Michel Foucault et la consommation. Gouverner et séduire, Caen/París, EMS, pp. 99-125.

Barthes, R. (1970): Elementos de semiología, Madrid, Comunicación/Alberto Corazón.

Barthes, R. (1990): La aventura semiológica, Barcelona, Paidós.

Bateson, G. (1985): Pasos hacia una ecología de la mente, Buenos Aires, Ediciones Carlos Lohlé.

Baudrillard, J. (1974): Crítica de la economía política del signo, México, Siglo XXI.

Baudrillard, J. (1978a): El sistema de los objetos, México, Siglo XXI.

Baudrillard, J. (1978b): Cultura y simulacro, Barcelona, Kairós.

Baudrillard, J. (2011): La sociedad de consumo, Madrid, Siglo XXI.

Berger, A. A. (2011): "The Branded Self: On the Semiotics of Identity", The American Sociologist, 42(2), pp. 232-237.

Boltanski, L. y È. Chiapello (2002): El nuevo espíritu del capitalismo, Madrid, Akal.

Bourdieu, P. (2000): Cuestiones de sociología, Madrid, Itsmo.

Braverman, H. (1974): Labor and Monopoly Capital, Nueva York, Monthy Review Preds.

Brems, C., M. Temmerman, T. Graham y M. Broersma (2017): "Personal Branding on Twitter: How employed and freelance journalists stage themselves on social media", Digital Journalism, 5(4), pp. 443-459.

Bröckling, U. (2016): The Entrepreneurial Self: Fabricating a New Type of Subject, London, Sage.

Cabanas, E. y E. Illouz (2019): Happycracia: cómo la ciencia y la industria de la felicidad controlan nuestras vidas, Madrid, Paidós.

Candil Moreno, D. (2020): "Tecnologías de subjetivación del intraemprendedor: el caso de la consultoría", Revista Española de Sociología, 29(2), pp. 233-248.

Castel, R. (2010): El ascenso de las incertidumbres. Trabajo, protecciones, estatuto del individuo, Buenos Aires, Fondo de Cultura Económica.

Chicchi, F., E. Leonardi y S. Luccarelli (2018): Más allá del salario. Lógicas de la explotación, Madrid, Enclave de libros. 
Codeluppi, V. (2000): Lo spettacolo della merce. I luoghi del consumo dai passages a Disney World, Milán, Bompiani.

Curcio, R. (2005): La empresa total, Madrid, Traficantes de Sueños.

Du Gay, P. (2012): En elogio de la burocracia, Madrid, Siglo XXI.

Du Gay, P. (2019): Consumo e identidad en el trabajo, Madrid, CIS.

Ehrenberg, A. (2009): Le culte de la perfomance, París, Calmann-Lévy, $3^{\mathrm{a}}$ ed.

Ehrenreich, B. (2018): Causas naturales. Cómo nos matamos por vivir más, Madrid, Turner.

Escandell Vidal, M. V. (1993): Introducción a la pragmática., Barcelona. Anthropos.

Fernández Rodríguez, C. J. (2007): El discurso del management: tiempo y narración, Madrid, CIS.

Fernández Rodríguez, C. J. (2008). "Management y sociedad en la obra de Peter Drucker”, Revista Internacional de Sociología, 49, pp. 195-218.

Fernández Rodríguez, Carlos J. (2019). "Gerencialismo a la vasca: un análisis del discurso de la 'nueva cultura de empresa", Lan Harremanak, 41, pp. 231-259.

Florida, R. (2010). La clase creativa, Barcelona y Madrid, Paidós/Espasa.

Foucault, M. (2009): Nacimiento de la biopolítica Curso del Collège de France (1978-1979), Madrid, Akal.

Friedrich, S. et al. (2018): La sociedad del rendimiento. Cómo el neoliberalismo impregna nuestras vidas, Barcelona, Katakrak.

Fuller, R. (2015): "How Quantified-Self Will Redefine the Future of the Enterprise", Wired, https://www.wired.com/insights/2015/01/quantified-self-enterprise [Consulta: 01/10/2019].

Giddens, A. (2000): Modernidad e identidad del yo, Barcelona, Península.

Goffman, E. (1994): La presentación de la persona en la vida cotidiana, Buenos Aires, Amorrortu.

Grice, H. P. (1991): “Lógica y conversación”, en Valdés, L. La búsqueda del significado, Madrid, Tecnos, pp. 511-530.

Hall, S. (2011): “The Neo-liberal Revolution", Cultural Studies, 25(6), pp. 705-728.

Hall, S., M. Merlino (2011): La cultura y el poder. Conversaciones sobre los cultural studies, Buenos Aires Amorrortu.

Kang, K. (2013): Branding Pays: The Five-step System to Reinvent Your Personal Brand, Palo Alto, CA, Branding Pays Media.

Hacking, I. (1986): "Making Up People", in T. L. Heller, M. Sosna and D.E. Wellbery (eds) Reconstructing Individualism, Stanford, CA, Stanford University Press.

Hochschid, A. R. (2011): La mercantilización de la vida íntima. Apuntes de la casa y el trabajo, Madrid, Katz.

Ilieș, V. I. (2018): "Strategic Personal Branding for Students and Young Professionals", Cross-Cultural Management Journal, XX (1), pp. 43-51.

Illouz, E. (2014): El futuro del alma. La creación de estándares emocionales, Madrid y Barcelona, Katz/CCCB.

Illouz, E. (Ed.) (2019): Capitalismo, consumo y autenticidad: las emociones como mercancía, Madrid, Katz.

Jodelet, D. (1986): "La representación social: fenómeno, concepto y teoría", en Moscovici, S. (Ed.), Psicología Social II, Barcelona, Paidós, pp. 469-494.

Kucharska, W. y P. Mikołajczak (2018): "Personal branding of artists and art-designers: necessity or desire?”, Journal of Product \& Brand Management, 27(3), pp. 249-261. 
Laclau, E. (2004): "Estructura historia y lo político", en Butler, J., Laclau, E. y Zizek, S., Contingencia, hegemonía, universalidad, Buenos Aires, FCE.

Landa, M. I., G. Blázquez y C. Castro (2019): "Emprender como estilo de vida. La 'actitud' en las dinámicas laborales de los trabajadores del fitness y del entretenimiento infantil" (Córdoba, Argentina), Debats, 133(1), pp 27-45.

Laval, C. y P. Dardot (2013): La nueva razón del mundo. Ensayo sobre la sociedad neoliberal, Barcelona, Gedisa.

López Salas, S. y M. Urraco Solanilla (2018): "Creación y gestión del valor de marca personal: personal branding y adaptación al nuevo escenario laboral contemporáneo", Revista Internacional de Turismo, Empresa y Territorio, 2(2), pp. 29-48.

Luca, F. A., C. A. Ioan y C. Sasu (2015): “The Importance of the Professional Personal Brand. The Doctors' Personal Brand", Procedia Economics and Finance, 20, pp. 350-357.

McNally, D. y K. D. Speak (2002): Be Your Own Brand: A Breakthrough Formula for Standing Out from the Crowd, San Francisco, Berrett-Koehler Publishers.

Medina-Vicent, M. (2018): "Análisis de la evaluación y las prescripciones morales sobre el comportamiento de las mujeres líderes en la literatura managerial", Empiria, Revista de Metodología de Ciencias Sociales, 41, pp. 103-126.

Miller, P. y N. Rose (1990): “Governing economic life”, Economy and Society, 19(1), pp. $1-31$.

Montoya, P. y T. Vandehey (2003): The Brand Called You: Make Your Business Stand Out in a Crowded Marketplace, Corona del Mar, CA, Personal Branding Press.

Mould, O. (2018): Against Creativity. Everything you have been told about creativity is wrong, Londres, Verso.

Muñoz-Rodríguez, D. y J. A. Santos Ortega (2017): "Las cárceles del capital humano: trabajo y vidas precarias en la juventud universitaria", Recerca, 20, pp. 59-78.

Nolan, L. (2015): "The impact of executive personal branding on non-profit perception and communications", Public Relations Review, 41, pp. 288-292.

Oliveira-Martins, M. d' (2018): Arlie Russell Hochschid. Un camino al corazón de la sociología, Madrid, CIS.

Orleán, A. (1999): Le pouvoir de la finance, París, Odile Jacob.

Peters, T. (1997): “The Brand called you”, Fast Company, 10(10), p. 83.

Peters, T. (2000): 50 claves para hacer de usted una marca, Bilbao, Deusto.

Peters, T. (2002): El talento. Cómo identificarlo y mantenerlo, Madrid, Nowtilus.

Pink, D. H. (2001): Free Agent Nation. The Future of Working for Yourself, Nueva York, Warner Books.

Rein, I., P. Kotler, M. Hamlin y M. Stoller (2006): High visibility: Transforming Your Personal and Professional Brand, Nueva York, McGraw-Hill.

Revilla Castro, J. C. (2016): "Reconstrucciones de la identidad laboral en contextos organizacionales invivibles", Politica y Sociedad, 54(1), pp. 65-86.

Ries, A. y J. Trout (2011): Positioning, The battle for your mind, Nueva York, McGrawHill Inc.

Roffer, R. F. (2002): Make a Name for Yourself: Eight Steps Every Woman Needs to Create a Personal Brand Strategy for Success, Nueva York, Broadway Books.

Rose, N. (1999): Powers of Freedom: Reframing Political Thought. Cambridge University Press, Cambridge.

Searle, J. (1986): Actos de habla, Madrid, Cátedra. 
Schawbel, D. (2009): Me 2.0: 4 Steps to Building Your Future, Nueva York, Diversion Books.

Schultz, M., M. Hatch y M. Larsen (Eds.) (2000): The Expressive Organization, Oxford, Oxford University Press.

Serrano Pascual, A. (2016): "Colonización política de los imaginarios del trabajo: la intervención paradójica del 'emprendedor", en Gil Calvo, E. (Coord.), Sociólogos contra el economicismo, pp. 110-128.

Smith, A. (1994): La riqueza de las naciones, Madrid, Alianza.

Vallas, S. y A. Christin (2018): "Work and Identity in an Era of Precarious Employment: How Workers Respond to 'Personal Branding' Discourse", Work and Occupations 45(1), pp. 3-37.

VV.AA. (2011): Personal Branding... hacia la excelencia y la empleabilidad en la marca personal, Madrid, Madrid Excelente.

Watzlawick, P., J. Beavin y D. D. Jackson (1971): Teoría de la comunicación humana, Buenos Aires, Tiempo Contemporáneo.

Weber, M. (2012): “Ensayos sobre sociología de la religión”, en Sociología de la religión, Madrid Akal.

Wee, L. y A. Brooks (2010): "Personal branding and the commodification of reflexivity", Cultural Sociology 4(1), pp. 45-62.

Zafra, R. (2017): El entusiasmo. Precariedad y trabajo creativo en la era digital, Barcelona, Anagrama.

Zarifian, P. (2001): Temps et modernité. Le Temps comme enjeu du monde moderne, París, L'Harmattan. 